\title{
An Enhanced-Time Difference of Arrival Technique for Estimating Mobile Station Position in Wireless Sensor Networks
}

\author{
Adekunle A. Adeyelu \\ Department of Mathematics \& \\ Computer Science, Benue \\ State University, Makurdi
}

\author{
Onaji J. Onah \\ Department of Mathematics \& \\ Computer Science, Benue \\ State University, Makurdi
}

\author{
Iwuese J. Orban \\ Department of Mathematics \& \\ Computer Science, Benue \\ State University, Makurdi
}

\begin{abstract}
There have been continuous search several hundred years from now for techniques to track an object from a remote location given certain facts. One of such is locating a Mobile Station (MS) which is an object within a cellular network. Existing outdoor techniques to locate this MS require optimization both in terms of accuracy and latency. In this paper, an Enhanced Mobile Station Positioning (MSP) model for Wireless Sensor Networks was developed and its performance was appraised using accuracy and latency metrics in line with Time Difference of Arrival (TDOA) procedure. This model used the difference in arrival time of the signal received at four base stations (BS) positioned within the neighborhood of the Mobile Station (MS) to locate the MS. The TDOA forms a hyperbola on which the MS can be found. The mathematical model was derived by solving the hyperbolas with Taylor's series expansion formula. The estimated position of the MS was calculated using Linear Least Square (LLS) solution in a repetitive manner. The performance of the formulated model was evaluated using accuracy and latency metrics. The result showed that the model located the MS within error distances of $189 \mathrm{~m}$ for $67 \%$ and $300 \mathrm{~m}$ for $95 \%$ of the time it was deployed. This result outclassed the same technique using three BS which located the MS within $235 \mathrm{~m}$ at $67 \%$ deployment and $349 \mathrm{~m}$ at $95 \%$ of the time the model was used. This gave approximately $14 \%$ improvement in accuracy. Simulation results also revealed that the latency experienced when the BSs were increased from three to four increased by $42.86 \%$ ( 0.085 seconds). It can be concluded that increasing the number of BSs from three to four gave a significant better accuracy in locating a MS within the BSs.
\end{abstract}

\section{General Terms}

Mobile Station Positioning techniques, GSM localization

\section{Keywords}

Mobile Station, Base Station, Time Difference of Arrival.

\section{INTRODUCTION}

Digital communication has evolved pretty well within the last few decades due to the increasing deployment of Wireless Sensor Networks. No doubt it has been a radical pattern shift that enabled multimedia communications between people and devices from any position. Mobile wireless communication system as an example of Cellular communication is realized using Global System for Mobile communications (GSM) devices. This GSM as effective and efficient as it is challenged with high energy consumption, security, screen size, storage capacity among others. Another major challenge is getting the exact or estimated location of an MS within a network (MSP).

For several hundred years now, methods to find the position of a remote object with respect to a known location have been required. In [1] it was reported that these methods were first applied in road surveying, target ranging for weaponry as well as map making. There has been a speedy boom in the number of MS users within the last decade, as a result, the number of applications that require location information is growing swiftly and MSP services has become a key research topic among researchers in the area [2].

Applications that render services based on the knowledge of the MS position are called Location Based Service (LBS). MSP techniques are therefore the backbone upon which LBS thrives. The original reason for the development of MSP techniques to support LBS in cellular networks was the need to trace the origin of emergency calls (E-911) [3]. At present, this initiative is now being applied in different areas, for example location sensitive billing, location based marketing, location of nodes in a distributed sensor network, intelligent traffic system, weather applications, military artillery, just to mention a few.

Many single techniques have been proposed to estimate location of MS. Examples include Global Positioning System (GPS), Angle of Arrival (AOA), Received Signal Strength (RSS), Time of Arrival (TOA), Time Difference of Arrival (TDOA), Signal Attenuation Difference of Arrival (SADOA), Cell Identification methods (Cell-ID). Other techniques which are hybrid include RSS/TDOA, TOA/AOA, AOA/TDOA, SADOA/TDOA among others. They combine their individual strengths to make up a hybrid which outperforms each constituent technique. Since this is the case, it follows that the quality of a hybrid technique depends on the quality of its constituent techniques. This has motivated the authors in this study to enhance the TDOA.

The Federal Communications Commission (FCC) of the United States of America issued out its accuracy standard in 2001 compelling all MSP service providers worldwide to work towards achieving it. The FCC accuracy requirement states that $67 \%$ of readings produced by any given (outdoor) MSP technique should be within 100 meters of the MS and $95 \%$ of the readings within 300 meters of the MS [4], [2]. The aim of every MSP technique therefore is to meet the FCC accuracy standard without necessarily consuming too much infrastructure or network resources in the process. The challenge lies in the fact that the accuracy of the existing (Network-based) outdoor positioning techniques is insufficient as they have not met the accuracy requirement of the FCC especially under the Non Line of Sight (NLOS) environments. Efforts are still being made in this regard. The 
TDOA technique is the leading individual MSP technique and produces superior performance when the BS is increased [5]. This study therefore proposes an enhanced TDOA model to locate a MS within a Wireless Sensor Network by improving on the work done by [2] which used 3 BS. This study made use of measurements from four BSs to improve accuracy. Each TDOA measurement between any two BS produces a hyperbola on which the MS may be found. It is common knowledge that an increment in BSs would definitely add to complexity in the algorithm and increase latency. This paper therefore investigated the latency caused by the additional BS to see if the solution is sustainable. The rest of the paper is as follows. Section 2 discusses some key related works by other authors, in section 3, the TDOA mathematical model is derived, the methodology for the model is described in detail, and the Algorithm is shown. In section 4, the accuracy and latency results are presented and discussed. Based on these results, conclusions are made in 5. The paper closes by suggesting future works in section 6 .

\section{LITERATURE REVIEW}

Due to the dramatic boom in MS usage within the last decade, MSP in cellular networks has become a hot topic among researchers. Some notable contributions by these authors are reviewed in this section.

Object location tracking started with the combination of the GPS technology with the internet. This made outdoor localization achieve good success in map navigation, people location, object tracking, etc. However, GPS technology meets its frustration in indoor applications because of the great weakening of the satellite signal and multipath effect caused by the obstruction from buildings and the complex indoor.

The two different approaches in position estimation techniques were presented in [6]: single estimation techniques and hybrid schemes. The single techniques include AOA, TDOA, TOA and RSS. The hybrid schemes for position estimation presented in the literature includes TOA/RSS, TDOA/RSS, TDOA/AOA and AOA/TOA. The literature showed that hybrid schemes tend to outperform single schemes. However, the more accurate a constituent technique of a hybrid is, the more accurate the hybrid will be. [7] In their works used Kalman Filters algorithm to locate a player within a football field. In the literature, TDOA and Kalfman Filters algorithm was combined to improve accuracy.

TDOA technique was studied in [8] and [5]. Specifically, the authors studied localization accuracy using Time Difference of Arrival (TDOA) measurements within a sensor network. Their results confirm high accuracy and show the superior performance of an additional BS. Attempts to grow accuracy led to the advent of many hybrid techniques which TDOA is a constituent part. [2] is an example of such a study. The authors proposed a RSS/TDOA location scheme using three BSs. According to their model the strength of the signal received at the $\mathrm{BS}$, is used to find the distance between the MS and BSs. The time difference of arrival of signal exchanged between the MS and the BSs forms a hyperbola on which the MS lies. Solving the intersection of the hyperbolas gives the location of the MS. The accuracy of the TDOA meets its downfall due to obstacles or complex propagation mechanisms. Hence the need for more efforts to improve on TDOA. According to [2], and [6] increasing the number of BSs would be even more beneficial in terms of accuracy. This paper therefore studies the accuracy of the TDOA taking measurements from 4 BS (instead of the conventional 3 BS) and further investigates the latency issues that arises due to additional BS.

\section{TIME DIFFERENCE OF ARRIVAL}

\subsection{Mathematical Model}

Suppose that each $\mathrm{BS}_{\mathrm{i}}$ is capable of performing TOA observation, $t_{i}$, then TDOA observation is defined as ,

$\mathrm{T}_{\mathrm{i}}=\mathrm{t}_{\mathrm{i}}-\mathrm{t}_{1}, \mathrm{i}=1, \ldots, \mathrm{N}$. Expressing TDOA observation as a function of station coordinates, the hyperbola produced is given by [2]

$c T_{i}=\sqrt{\left(x-x_{i}\right)^{2}+\left(y-y_{i}\right)^{2}}-\sqrt{\left(x-x_{1}\right)^{2}+\left(y-y_{1}\right)^{2}}$

is obtained. Where $\left(x_{1}, y_{1}\right)$ and $\left(x_{i}, y_{i}\right)$ are the coordinates of $\mathrm{BS}_{1}$ and $\mathrm{BS}_{\mathrm{i}}$, respectively, and $(x, y)$ is the unknown MS position. Consequently, the MS position is determined by solving the intersections of a set of $N-1$ hyperbola

Denote the initial guess of the MS position as $\left(x_{0}, y_{0}\right)$, the LLS solution is a common technique used to solve TDOA equations by using the first two terms of their Taylor series.

where:

$M(x, y)=$

$\sqrt{\left(x-x_{i}\right)^{2}+\left(y-y_{i}\right)^{2}}-\sqrt{\left(x-x_{1}\right)^{2}+\left(y-y_{1}\right)^{2}}$

$-c T_{i}=0$

The Taylor series expansion of $M(x, y)$ around the point $\left(x_{0}, y_{0}\right)$ is given by

$M(x, y)=M\left(x_{0}, y_{0}\right)+\frac{\partial \mathrm{M}(x, y)}{\partial x} \downarrow_{x=x_{0}}\left(x-x_{0}\right)+$

$\frac{\partial \mathrm{M}(x, y)}{\partial y} \downarrow_{y=y_{0}}\left(y-y_{0}\right)+$ Higher Terms

Therefore;

$$
\begin{aligned}
& M\left(x_{0}, y_{0}\right)=\sqrt{\left(x_{0}-x_{i}\right)^{2}+\left(y_{0}-y_{i}\right)^{2}}- \\
& \sqrt{\left(x_{0}-x_{1}\right)^{2}+\left(y_{0}-y_{1}\right)^{2}}-c T_{i} \\
& \frac{\partial \mathrm{M}(x, y)}{\partial x} \downarrow_{x=x_{0}}\left(x-x_{0}\right)= \\
& {\left[\frac{\left(x-x_{i}\right)}{\sqrt{\left(x_{0}-x_{i}\right)^{2}+\left(y_{0}-y_{i}\right)^{2}}}-\frac{\left(x_{0}-x_{1}\right)}{\sqrt{\left(x_{0}-x_{1}\right)^{2}+\left(y_{0}-y_{1}\right)^{2}}}\right]} \\
& \left(x-x_{0}\right) \frac{\partial \mathrm{M}(x, y)}{\partial y} \downarrow_{y=y_{0}}\left(y-y_{0}\right)= \\
& {\left[\frac{\left(y_{0}-y_{i}\right)}{\sqrt{\left(x_{0}-x_{i}\right)^{2}+\left(y_{0}-y_{i}\right)^{2}}}-\frac{\left(y_{0}-y_{1}\right)}{\sqrt{\left(x_{0}-x_{1}\right)^{2}+\left(y_{0}-y_{1}\right)^{2}}}\right]\left(y-y_{0}\right)}
\end{aligned}
$$

This gives;

$$
M(x, y)=
$$$$
\sqrt{\left(x_{0}-x_{i}\right)^{2}+\left(y_{0}-y_{i}\right)^{2}}-\sqrt{\left(x_{0}-x_{1}\right)^{2}+\left(y_{0}-y_{1}\right)^{2}}-c T_{i}
$$ 


$$
\begin{gathered}
{\left[\frac{\left(x-x_{i}\right)}{\sqrt{\left(x_{0}-x_{i}\right)^{2}+\left(y_{0}-y_{i}\right)^{2}}}-\frac{\left(x_{0}-x_{1}\right)}{\sqrt{\left(x_{0}-x_{1}\right)^{2}+\left(y_{0}-y_{1}\right)^{2}}}\right]\left(x-x_{0}\right)} \\
+ \\
{\left[\frac{\left(y_{0}-y_{i}\right)}{\sqrt{\left(x_{0}-x_{i}\right)^{2}+\left(y_{0}-y_{i}\right)^{2}}}-\frac{\left(y_{0}-y_{1}\right)}{\sqrt{\left(x_{0}-x_{1}\right)^{2}+\left(y_{0}-y_{1}\right)^{2}}}\right]\left(y-y_{0}\right)=0}
\end{gathered}
$$

where

$$
m_{x i}=\frac{\left(x-x_{i}\right)}{\sqrt{\left(x_{0}-x_{i}\right)^{2}+\left(y_{0}-y_{i}\right)^{2}}}-\frac{\left(x_{0}-x_{1}\right)}{\sqrt{\left(x_{0}-x_{1}\right)^{2}+\left(y_{0}-y_{1}\right)^{2}}}
$$

$$
m_{y i}=\frac{\left(y_{0}-y_{i}\right)}{\sqrt{\left(x_{0}-x_{i}\right)^{2}+\left(y_{0}-y_{i}\right)^{2}}}-\frac{\left(y_{0}-y_{1}\right)}{\sqrt{\left(x_{0}-x_{1}\right)^{2}+\left(y_{0}-y_{1}\right)^{2}}}
$$

$$
f_{i}=\sqrt{\left(x_{0}-x_{i}\right)^{2}+\left(y_{0}-y_{i}\right)^{2}}-\sqrt{\left(x_{0}-x_{1}\right)^{2}+\left(y_{0}-y_{1}\right)^{2}}
$$

After expanding (3) and substituting

$m_{x i} x+m_{y i} y=c T_{i}-f_{i}+m_{x i} x_{0}+m_{y i} y_{0}$

Expressing the set of linear equations in (4) in matrix form, it yields a solution of the form

$$
\mathrm{A} \hat{X}_{\mathrm{MS}}=\mathrm{B}
$$

where

$$
\begin{aligned}
& \mathrm{A}=\left[\begin{array}{ll}
m_{x 2} & m_{y 2} \\
m_{x 3} & m_{y 3} \\
m_{x 4} & m_{y 4}
\end{array}\right], \quad \hat{X}_{\mathrm{MS}}=\left[\begin{array}{l}
x \\
y
\end{array}\right], \\
& \mathrm{B}=\left[\begin{array}{l}
c T_{2}-f_{2}+m_{x 2} x_{0}+m_{y 2} y_{0} \\
c T_{3}-f_{3}+m_{x 3} x_{0}+m_{y 3} y_{0} \\
c T_{4}-f_{4}+m_{x 4} x_{0}+m_{y 4} y_{0}
\end{array}\right]
\end{aligned}
$$

The LLS solution is gotten from

$\hat{X}_{\mathrm{MS}}=\left(A^{T} A\right)^{-1} A^{T} \mathrm{~B}$

After the LLS solution is performed repeatedly by solving hyperbolas about the point $\hat{X}_{\mathrm{MS}}$. The current $\hat{X}_{\mathrm{MS}}$ estimate is fed back into the new estimate to be calculated until any further estimate would no longer yield a significant result but most likely waste time.

\subsection{Methodology}

This section describes how the simulation was done. A software package called Matlab was used for the simulation. Fig 2 shows that areas on the Cartesian plane were divided into hexagonal cells. Four arbitrary points $\left(x_{1}, y_{1}\right),\left(x_{2}, y_{2}\right),\left(x_{3}, y_{3}\right)$ and $\left(x_{4}, y_{4}\right)$ were chosen to represent the various BS coordinates, $B S_{1}, B S_{2}, B S_{3}$ and $B S_{4}$. The MS was placed in a known initial position $\left(x_{0}, y_{0}\right)$ between the 4 BSs. The exact distances from the MS position to the various BS was also calculated and are represented as D1, D2, D3 and D4 respectively. A zero mean Gaussian random variable is generated as the noise (in decibel per metre) and subtracted from the value of the signal strength received at each BS. This noise value further varies the distance calculated between the MS and each BS coordinate. This would yield new distance values D11, D22, D33 and $\mathrm{D} 44$. If the time to travel from the MS to $\mathrm{BS}_{1}$ and from the MS to $\mathrm{BS}_{2}$ is $t_{1}$ and $t_{2}$ respectively, it follows that $t 1-t 2$ is a constant. This means the difference between the corresponding distances D11 and D22 is also constant. Therefore the MS in-between the two BSs travels along a hyperbola and can be located on this hyperbola. See Fig 1 . The TDOA using 4 BS produces three hyperbolas and the point of intersection between these hyperbolas produces the initial estimate for the MS position. The LLS solution is deployed to solve the matrices resulting from the linearization of the TDOA technique. The location performance was assessed in terms of the value of the distance error (Root Mean Square Error) defined by:

$E_{d}=\sqrt{\left(x_{M S}-\hat{x}_{M S}\right)^{2}+\left(y_{M S}-\hat{y}_{M S}\right)^{2}}$

where $\left(x_{M S}, y_{M S}\right)$ and $\left(\hat{x}_{M S}, \hat{y}_{M S}\right)$ are the actual and estimated MS locations, respectively [2]. After several free runs of TDOA location estimation for random noise values between MS and BS, results are presented in section 4.

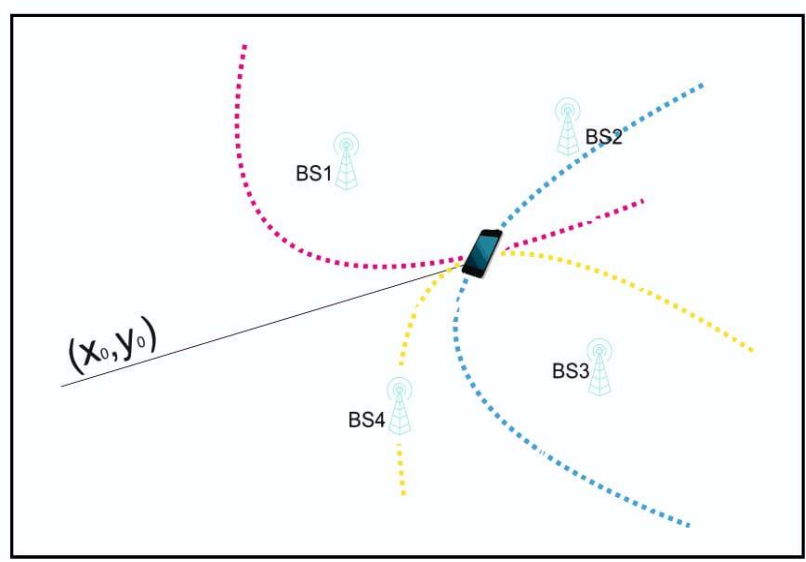

Fig 1: Time Difference of Arrival 


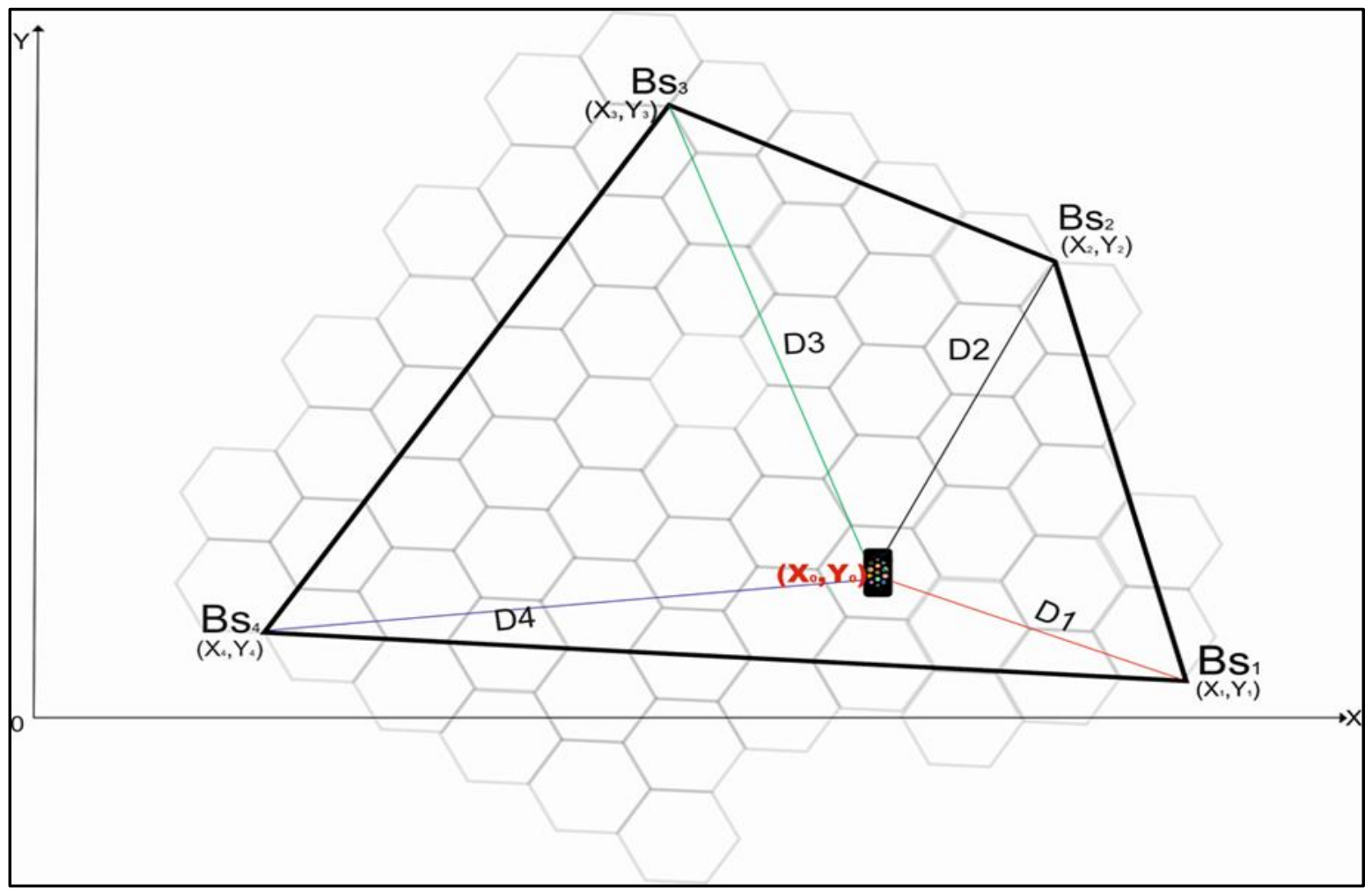

Fig 2: Simulation of the system

\subsection{Algorithm of the System}

The algorithm of the TDOA model is given in this section. Some assumptions were made as stated.

\subsubsection{Overall Algorithm of the System}

PRO: $x_{n}, y_{n}$ are the coordinates of the $n^{\text {th }}$ base station. The phone to be located is assumed to be a Mobile Phone object (MS) with the following properties. MS identity (imsi), Serving base station of the MS (sbs), a list of neighboring base stations $\left(\mathrm{nbs}_{\mathrm{i}, \mathrm{j}}\right)$ where $1<=\mathrm{i}<=6$ and $1<=\mathrm{j}<=2$, a list of neighboring sorted base stations $\left(\mathrm{nsbs}_{\mathrm{i}, \mathrm{j}}\right)$ where $1<=\mathrm{i}<=4$ and $1<=\mathrm{j}<=2$, The base stations have the following nested properties: signal strength, Base Station Code (cid), latitude (x), longitude (y).

POST: This algorithm calculates the location of a Mobile Station $\mathrm{X}_{\mathrm{ms}}$ using the parameters in PRO and the formula in equation 12.

Step 1: Identify the calling Mobile Station Object (MS)

Step 2: Get MS.sbs

Step 3: Set $\mathrm{i}=1$

Step 4: Repeat step 5 to 7 while $\mathrm{i}<=6$

Step 5: set nbs $[i, 1]=$

getSignalStrengthDBM(MS.getNextnbs());

Step 6: set nbs[i,2] = getCID(MS.getNextnbs ()$)$;

Step 7: set $\mathrm{i}=\mathrm{i}+1$

Step 8: sort nbs with the bubblesort algorithm (in descending order) as in 3.3.2

Step 9: nsbs[1,1] = getSignalStrengthDBM(MS.sbs);

Step 10: nsbs[1,2] = getCID(MS.sbs);

Step 11: set i to 2

Step 12: Repeat step 13 to 15 while $\mathrm{i}<4$

Step 13: set nsbs[i,1] = get nbs[i-1,1]

Step 14: set nsbs[i,2] = get nbs[i-1,2]

Step 15: set $\mathrm{i}=\mathrm{i}+1$

Step 16: feed the content of nsbs into equation 6 to produce $\mathrm{X}_{\mathrm{ms}}$
Step 17: Exit

\subsubsection{Bubble Sort Algorithm (Descending Order)}

PRO: Assume list is an unsorted array of $\mathrm{n}$ elements.

POST: This algorithm returns the sorted version of list in descending order.

Step 1: Start

Step 2: for all elements of list Repeat step 3 to 6

Step 3: if list $[\mathrm{i}]<\operatorname{list}[\mathrm{i}+1]$ Repeat step 4 to 6

Step 4: temp = list $[\mathrm{i}]$;

Step 5: list $[\mathrm{i}]=$ list $[\mathrm{i}+1]$;

Step 6: list $[i+1]=$ temp;

Step 7: Exit

\section{RESULT AND DISCUSSION}

\subsection{Simulation Data}

The various $E_{d}$ calculated for each iteration was expressed as a percentage of the Cumulative Distribution Function (CDF) and labelled as Location Distance. The result is presented in Table 1 and Table 2 below.

Table 1. TDOA 3 BS data set

\begin{tabular}{|l|l|}
\hline \multicolumn{2}{|c|}{ TDOA 3 BS } \\
\hline $\begin{array}{l}\text { Location } \\
\text { Distance(m) }\end{array}$ & CDF (\%) \\
\hline 20.45 & 0.008752 \\
\hline 23.10 & 0.069536 \\
\hline 24.50 & 0.157772 \\
\hline 25.50 & 0.265615 \\
\hline 26.20 & 0.387184 \\
\hline 34.00 & 0.661693 \\
\hline 39.40 & 1.042085 \\
\hline 42.80 & 1.489144 \\
\hline 45.50 & 1.989144 \\
\hline
\end{tabular}




\begin{tabular}{|l|l|}
\hline 49.50 & 2.567576 \\
\hline 54.60 & 3.246007 \\
\hline 56.50 & 3.961693 \\
\hline 61.40 & 4.773458 \\
\hline 61.90 & 5.595027 \\
\hline 65.70 & 6.491105 \\
\hline 69.10 & 7.453850 \\
\hline 73.90 & 8.510713 \\
\hline 75.00 & 9.589144 \\
\hline 86.70 & 10.89699 \\
\hline 93.30 & 12.33424 \\
\hline 94.50 & 13.79503 \\
\hline 94.70 & 15.25973 \\
\hline 95.20 & 16.73424 \\
\hline 97.90 & 18.26169 \\
\hline 105.30 & 19.93424 \\
\hline 106.30 & 21.62640 \\
\hline 107.30 & 23.33816 \\
\hline 112.10 & 25.14405 \\
\hline 120.20 & 27.10875 \\
\hline 133.30 & 29.33032 \\
\hline 136.10 & 31.60679 \\
\hline 139.20 & 33.94405 \\
\hline 139.90 & 36.29503 \\
\hline 140.50 & 38.65777 \\
\hline 153.80 & 41.28130 \\
\hline 154.50 & 43.91856 \\
\hline 169.90 & 46.85777 \\
\hline 197.40 & 50.33620 \\
\hline 203.50 & 53.93424 \\
\hline 206.70 & 57.59503 \\
\hline 225.70 & 61.62836 \\
\hline 234.60 & 65.83620 \\
\hline 234.80 & 70.04797 \\
\hline 237.40 & 74.31071 \\
\hline 244.80 & 78.71856 \\
\hline 251.50 & 83.25777 \\
\hline 274.90 & 88.25581 \\
\hline 310.80 & 100.49700 \\
\hline 353.50 & 112.21660 \\
\hline 517.70 & \\
\hline & \\
\hline & \\
\hline
\end{tabular}

Table 2. TDOA 4 BS data set

\begin{tabular}{|l|r|}
\hline \multicolumn{2}{|c|}{ TDOA 4 BS } \\
\hline $\begin{array}{l}\text { Location } \\
\text { Distance(m) }\end{array}$ & CDF (\%) \\
\hline 10.45 & 0.008752 \\
\hline 13.10 & 0.069536 \\
\hline 14.50 & 0.157772 \\
\hline 15.50 & 0.265615 \\
\hline 16.20 & 0.387184 \\
\hline 17.00 & 0.661693 \\
\hline 19.40 & 1.042085 \\
\hline 22.80 & 1.489144 \\
\hline
\end{tabular}

\begin{tabular}{|c|c|}
\hline 35.50 & 5.989144 \\
\hline 39.50 & 8.567576 \\
\hline 44.60 & 12.24601 \\
\hline 46.50 & 12.96169 \\
\hline 51.40 & 13.77346 \\
\hline 51.90 & 13.59503 \\
\hline 55.70 & 15.49111 \\
\hline 59.10 & 17.45385 \\
\hline 63.90 & 17.51071 \\
\hline 65.00 & 19.58914 \\
\hline 76.70 & 19.89699 \\
\hline 83.30 & 21.33424 \\
\hline 84.50 & 22.79503 \\
\hline 84.70 & 23.25973 \\
\hline 85.20 & 25.73424 \\
\hline 87.90 & 26.26169 \\
\hline 95.30 & 27.10875 \\
\hline 96.30 & 29.33032 \\
\hline 97.30 & 31.60679 \\
\hline 102.10 & 33.94405 \\
\hline 110.20 & 36.29503 \\
\hline 123.30 & 38.65777 \\
\hline 126.10 & 41.28130 \\
\hline 129.20 & 43.91856 \\
\hline 129.90 & 44.03990 \\
\hline 130.50 & 44.29112 \\
\hline 132.80 & 45.23543 \\
\hline 135.10 & 46.09888 \\
\hline 137.40 & 46.85777 \\
\hline 139.70 & 50.33620 \\
\hline 143.00 & 53.93424 \\
\hline 158.00 & 57.59503 \\
\hline 165.00 & 61.62836 \\
\hline 172.00 & 65.83620 \\
\hline 189.50 & 67.04797 \\
\hline 206.70 & 74.31071 \\
\hline 209.70 & 78.71856 \\
\hline 219.50 & 83.25777 \\
\hline 225.90 & 88.25581 \\
\hline 259.00 & 93.45433 \\
\hline 300.80 & 95.95777 \\
\hline 343.50 & 100.49700 \\
\hline 607.70 & 112.21660 \\
\hline
\end{tabular}

\subsubsection{Accuracy Result}

In this section, the dataset in Table 1 and Table 2 were plotted for easy analysis. The performance of each technique is analyzed based on the CDF of distance error (location distance) mainly at $67 \%$ and $95 \%$. This result reveals by how much this solution approached the FCC accuracy requirement. 


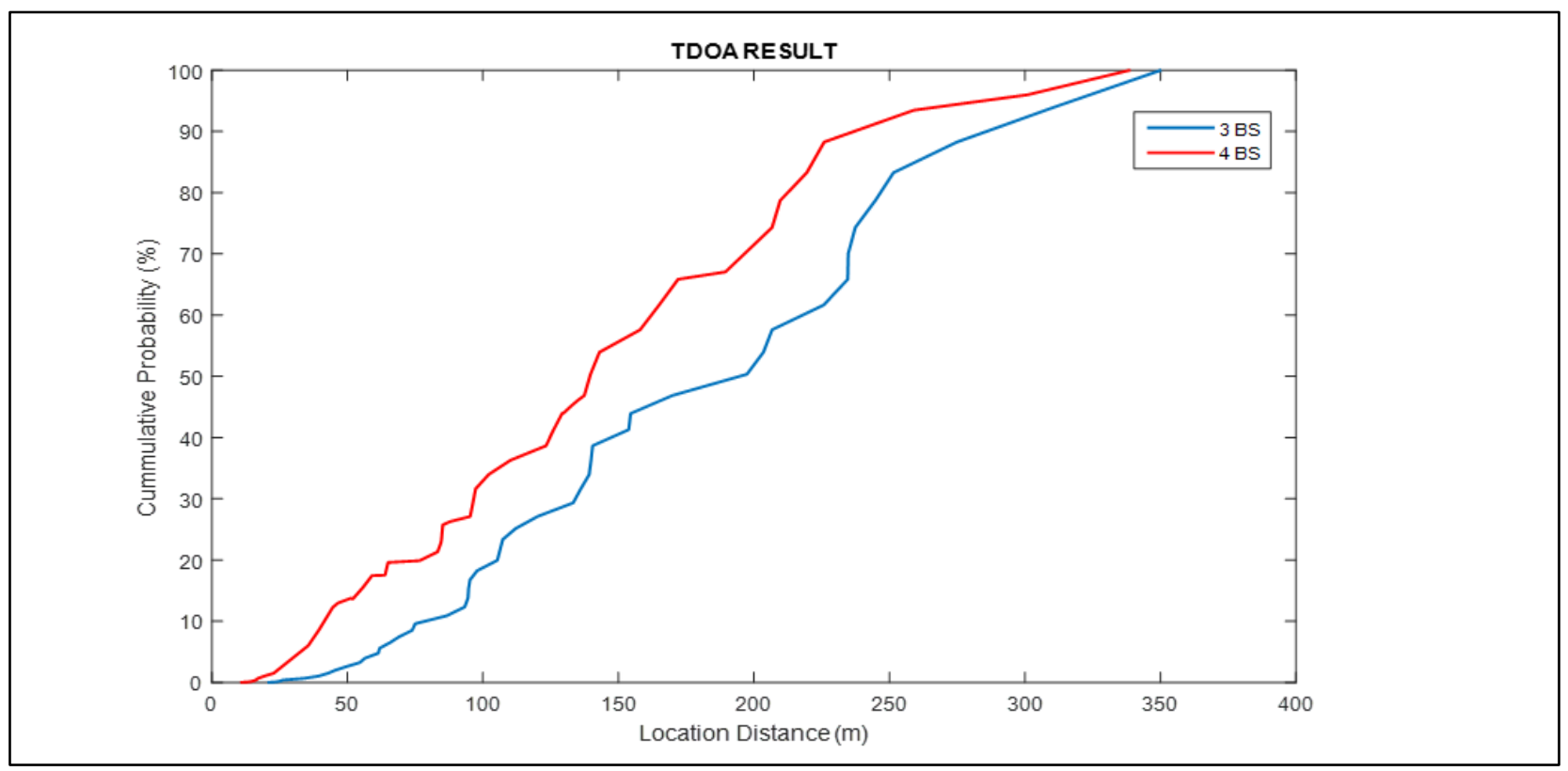

Fig 2: TDOA graph for 3 and 4 BSs

Fig 2 shows that with readings from 3 BS, at $67 \%$ the MS was located within a distance of approximately $235 \mathrm{~m}$. While at the confidence level of $95 \%$, MS was found within $349 \mathrm{~m}$. In the case of 4 BS however, the MS location distance was $189 \mathrm{~m}$ at $67 \%$ deployment, and $300 \mathrm{~m}$ at $95 \%$ of use.

Table 3. Accuracy Result Summary

\begin{tabular}{|c|c|c|c|}
\hline $\begin{array}{c}\text { CDF } \\
(\%)\end{array}$ & $\begin{array}{c}\text { TDOA } \\
\text { METHOD }\end{array}$ & $\begin{array}{c}\text { Location } \\
\text { Distance(m) }\end{array}$ & $\begin{array}{c}\text { Enhancement } \\
\text { Level (\%) }\end{array}$ \\
\hline \multirow{2}{*}{67} & 3 BS & 235 & \multirow{2}{*}{19.6} \\
\cline { 2 - 3 } & $\begin{array}{c}4 \text { BS } \\
\text { (Enhanced) }\end{array}$ & 189 & \multirow{2}{*}{14.0} \\
\hline \multirow{2}{*}{95} & 3 BS & 349 & \multirow{2}{*}{14.0} \\
\cline { 2 - 3 } & $\begin{array}{c}4 \text { BS } \\
\text { (Enhanced) }\end{array}$ & 300 \\
\hline
\end{tabular}

As shown in Table 3, the Enhanced technique improved on the 3 BS case by $19.6 \%$ and $14 \%$ at deployment levels of $67 \%$ and $95 \%$ respectively. This delivered an overall $16.8 \%$ improvement of the Enhanced TDOA over its 3 BS counterpart.

\subsubsection{Latency Result}

In this section, the average time it took to produce the location estimate for each technique is displayed. Latency is the delay experienced when producing a location estimate. In this case, the extra time delay experienced when the BS was increased from 3 to 4 gives the latency. A very high latency value could render a solution insignificant or totally unworkable notwithstanding a significant increase in accuracy. See Table 5 for comparison.

Table 5. Latency Result

\begin{tabular}{|c|c|c|}
\hline TDOA METHOD & 3 BS & 4 BS (Enhanced) \\
\hline $\begin{array}{c}\text { Average Elapsed } \\
\text { Time (Seconds) }\end{array}$ & 0.113322 & 0.0 .198322 \\
\hline Latency Value & \multicolumn{2}{|c|}{0.085} \\
\hline Percentage Latency & \multicolumn{2}{|c|}{$42.86 \%$} \\
\hline
\end{tabular}

As shown in Table 5, the latency value is 0.085 seconds which makes a $42.86 \%$ increment in time from 3 to 4 BS.

\section{CONCLUSION}

In this study, an enhanced TDOA technique was used to efficiently find the position of a MS in urban and suburban areas. The latency issues that accompanied the increase in number of BS from the conventional 3 to 4 was also investigated. This study showed that the developed technique with 4 BS outperformed the same technique using 3 BS given that no hardware adjustments were made to the currently available handsets. Simulation results showed that $67 \%$ of the readings were within 189 meters of the MS and $95 \%$ of the readings were within 300 meters. Hence, there is up to $16.8 \%$ improvement on the developed technique (using 4 BS) over the same technique using $3 \mathrm{BS}$.

Furthermore, the latency value was 0.085 seconds. Efforts could still be made to reduce this latency while sustaining the accuracy. The solution presented in this work is valuable to Communication Engineers, football league industry for goal line technology as well as any distress caller. The extra computation loading at the network end can be calmed by using modern powerful computation machines.

\section{FUTURE WORKS}

The following is recommended for future studies on this work:

i. Efforts should be made to reduce latency without compromising accuracy.

ii. Developed techniques should be stretched to locate more than one MS at a time. This would actually improve latency.

iii. More than two techniques could be merged and their performance evaluated.

iv. Other techniques could be employed to perhaps meet the FCC accuracy requirement.

\section{ACKNOWLEDGMENTS}

Our thanks to the Almighty God who has been by our side all 
through this research. His grace was sufficient to start and finish this work.

\section{REFERENCES}

[1] T. B. Timothy, H. B. Huub, A. M. Ken and H. P. Wyatt, "A Review of Position Tracking Methods," 1st International Conference on Sensing Technology, p. 1, 2012.

[2] A.-R. Sharief, K. Yahya and A.-I. Mohammad, "Mobile Station Positioning using Time Difference of Arrival and Received Signal Strength," International Journal of Mobile Communications, October 2012.

[3] R. S. Campos, "Evolution of Positioning Techniques in Cellular Networks, from 2G to 4G," Wireless Communications and Mobile Computing, p. 1, 12 January 2017.

[4] J. A. d. Peral-Rosado, R. Raulefs, J. A. L'opez-Salcedo and G. Seco-Granados, "Survey of Cellular Mobile Radio Localization Methods: from 1G to 5G," IEEE Communications Surveys and Tutorials, pp. 9-11, 2017.

[5] R. Kaune, J. Hörst and W. Koch, "Accuracy analysis for TDOA localization in sensor networks," 14th International Conference on Information Fusion, 5-8 July 2011.
[6] A. D. Gante and M. Siller, "A Survey of Hybrid Schemes for Location Estimation in Wireless Sensor Networks," The 2013 Iberoamerican Conference on Electronics Engineering and Computer Science, 2013.

[7] S. Rangarajan, M. Kanduri, V. Kumar and R. Medapati, "Evaluation of TDOA based Football Player's Position Tracking Algorithm using Kalman Filter," june 2018.

[8] R. Kaune, "Accuracy Studies for TDOA and TOA Localization," 15th International Conference on Information Fusion, 2012.

[9] H.-L. Song, "Automatic Vehicle Location in Cellular Communications Systems," IEEE TRANSACTIONS ON VEHICULAR TECHNOLOGY, vol. 43, no. 4, November 1994.

[10] C. Li, L. Mo and D. Zhang, "Review on UHF RFID Localization methods," JRFID.2019.2924346, IEEE Journal, 2019.

[11] G. Kbar and W. Mansoor, "Mobile Station Location based on Hybrid of Signal Strength and Time of Arrival," Proceedings of the International Conference on Mobile Business, 2005. 